\title{
PROPIEDADES FÍSICAS Y SENSORIALES DE UN PAN FRESCO, CON LA ADICIÓN DE LAS ENZIMAS LACASA, XILANASA Y LIPASA
}

\author{
$\triangle$ ÓsCAR VEGA ${ }^{1}$ \\ RuBÉN DE MARCO ${ }^{2}$ \\ CECILIA Di RISIO ${ }^{3}$
}

\section{RESUMEN}

El objetivo de la presente investigación fue evaluar algunas propiedades físicas y sensoriales de un pan elaborado con la combinación de las enzimas lacasa, xilanasa y lipasa, con el fin de proponer un producto panificable sin aditivos químicos. La metodología incluyó la determinación del volumen, volumen específico y análisis de características internas del pan como color de miga según la Norma IRAM 15858-1. El análisis sensorial se realizó mediante una prueba triangular compuesta por 38 jueces no entrenados, las diferencias significativas de los resultados se analizaron mediante las tablas Bengtsson's con un nivel de significancia del 95 \%. Como resultado principal se obtuvo que el volumen de los panes obtenidos con las diferentes formulaciones varió entre $4,76 \mathrm{~cm}^{3}$ y 7,84 $\mathrm{cm}^{3}$, en tanto que el volumen especifico obtenido para la formulación de un pan compuesto por lacasa-xilanasa-lipasa fue de $5,23 \mathrm{~cm}^{3} / \mathrm{g}$. En cuanto al análisis sensorial, no reportó diferencias significativas la aceptabilidad del pan formulado en esta investigación versus un pan con aditivos químicos tradicionales. Se puede concluir que la combinación de las tres enzimas utilizadas dio un lugar a un panificado con características propias del producto.

PALABRAS CLAVE: pan, enzimas, lacasa, xilanasa, lipasa, propiedades físicas, análisis sensorial.

\section{PHYSICAL AND SENSORY PROPERTIES OF FRESH BREAD, WITH ADDITION OF ENZYMES LACCASE, XYLANASE AND LIPASE}

\section{ABSTRACT}

The aim of this investigation was to evaluate some physical and sensory properties of bread made with combination of different enzymes such as the Laccasa , Xylanase and Lipase, with the aim of proposing a bakery product without chemical additives. The methodology included the determination of Volume, Specific Volume, analysis of internal characteristics of bread and crumb color as the IRAM NORM 15858-1; sensory analysis was performed using a triangular test consisting of 38 untrained judges, the significant differences results were analyzed by Bengtsson's tables with a

1 Ingeniero Agrícola. MSc. Tecnología de Alimentos.

2 Licenciado en Química. Especialista en Micología de Alimentos. CBC. Universidad de Buenos Aires.

3 Licenciada en Ciencias Químicas. Doctora en Ciencias Químicas. Universidad de Buenos Aires.

Autor de correspondencia: Vega, O. (Óscar). Universidad de Antioquia. Grupo BIOALI, Departamento de Alimentos. Medellín, Colombia. Calle 67No.53-108, Bl 1-7 Off-237/ Tel.: (574) 2638282.

Correo electrónico: oscar.vega@udea.edu.co
Historia del artículo:

Artículo recibido: 28-X-2014 / Aprobado: 13-II-2015

Disponible online: 30 de enero de 2016

Discusión abierta hasta noviembre de 2016 
significance level of $95 \%$. The main results, that the volume of the bread obtained with different formulations ranged between $476 \mathrm{~cm}^{3}-784 \mathrm{~cm}^{3}$, while the specific volume obtained for the formulation of composed by xylanase - laccase lipase was $5.23 \mathrm{~cm}^{3} / \mathrm{g}$. As for the sensory analysis, there aren't significant differences reported the acceptability of the bread made in this research versus traditional bread with chemical additives. It can be concluded that the combination of the three enzymes used (laccasa, xylanase and lipase) gave rise to a baked good product with characteristics.

KEYWORDS: bread, laccase, xylanase, lipase, physical properties, sensory analysis.

\section{AS PROPRIEDADES FÍSICAS E SENSORIAIS DO PÃO FRESCO COM A ADIÇÃO DA ENZIMA LACASE, XILANASE E LIPASE}

\section{RESUMO}

O objetivo deste estudo foi avaliar algumas propriedades físicas e sensoriais de um pão feito com a combinação das enzimas lacase, xilanase e lipase, a fim de propor um produto de padaria, sem aditivos químicos. A metodologia incluiu a determinação do volume, o volume específico e análise de recursos internos, tais como cor do miolo de pão de acordo com a norma IRAM 15.858-1. A análise sensorial foi realizada utilizando um teste de triângulo composto por 38 juízes não treinados, as diferenças significativas nos resultados foram analisados por tabelas do Bengtsson com um nível de significância de 95\%. O principal resultado foi obtido, o volume dos pães obtidos com diferentes formulações variou entre 4,76 e $7,84 \mathrm{~cm}^{3}$, enquanto que o volume específico obtido para o desenvolvimento de um pão composto por lacase-xilanase - lipase foi de $5,23 \mathrm{~cm}^{3}$ / g. em quanto ao análise sensorial, não teve diferenças significativas a aceitabilidade de pão feito nesta pesquisa contra o tradicional pão com aditivos químicos. Pode concluir-se que a combinação das três enzimas utilizadas deu origem a um bom produto cozido com as suas próprias características.

PALAVRAS-CHAVE: pão, enzimas, lacase, xilanase, lipase, propiedades físicas, analise sensorial.

\section{INTRODUCCIÓN}

El pan es uno de los productos de mayor consumo en el mundo, siendo Alemania el país con mayor consumo con alrededor de $120 \mathrm{~kg}$ per cápita. Para el caso latinoamericano el consumo lo lidera Chile, con 96 kg per cápita y Colombia se ubica en el quinto lugar con $23 \mathrm{~kg}$ per cápita (Fenalco, 2013). Por otro lado, se ha evidenciado que en el país se usan aditivos prohibidos -como el bromato de potasio- en la elaboración del pan, convirtiendo a este alimento, en un potencial riesgo para la salud (Vega et al., 2010). Dado lo anterior y de su importancia para la alimentación, se hace necesario generar investigaciones que permitan obtener nuevos productos panificables a partir de nuevas materias primas o aditivos naturales, que favorezcan la salud del consumidor. La industria de alimentos está en una búsqueda constante de materias primas, ya que el consumidor busca alimentos saludables y con menos aditivos de origen químico, no siendo la industria de la panificación la excepción.

La tendencia en esta industria es el uso de nuevas harinas, enzimas o el diseño de panificables para celiacos, algunas de estos desarrollos son publicados por (Mahmoud et al., 2013; Perez et al., 2012; Gamonpilas et al., 2014; Van der Goot et al., 2011; Laureati et al., 2012; Korus et al., 2013; Korus et al., 2010), además de trabajos que buscan el remplazo de aditivos que han sido usados tradicionalmente, como el bromato de potasio, pero que son nocivos para la salud (Ribotta et al., 1999; Solito y Pavesi, 2003).

A nivel mundial se reportan estudios del uso de nuevas materias primas diferentes al trigo, para la elaboración de pan, además del uso de nuevos 
aditivos como son las enzimas, productos de carácter prebiótico, probiótico o con adición de vitaminas y minerales e incluso té verde (Chen et al., 2014; Batifouliera et al., 2005; Morais et al., 2013; Mihhalevski et al., 2013; Demigne et al., 2006; Rosell et al, 2012; Zhou et al., 2007).

Sin embargo, el uso de nuevas materias primas afecta las propiedades fisicoquímicas, sensoriales, texturales y reológicas de los productos panificables. En este sentido, diversos autores han determinado algunas de estas propiedades del pan en función de las materias primas usadas (Shittu et al., 2007; Abdelrahman et al., 2012; Bovell-Benjamin et al., 2008; Haros et al., 2013) reportan la valoración de propiedades tales como volumen, volumen especifico, además de determinación de parámetros reológicos, microbiológicos y micro-estructurales de panes elaborados a partir de harina de yuca, trigo, garbanzo, papa dulce y amaranto. En cuanto a los parámetros sensoriales, Mishra et al. (2012) y Arasaratnam et al. (2010) encontraron dichos parámetros en panes elaborados con harina de mijo, trigo y malta de arroz. Otros estudios en esta misma dirección evalúan propiedades físicas y sensoriales son reportados por Baiano et al. (2009); Vodovotz y Lodi (2008); Mandala (2005), Kim et al. (2013); Noor et al. (2013) y Arendt et al. (2010).

Por otro lado, la búsqueda de aditivos naturales o que no generen residuales en los alimentos ha llevado usar enzimas en el diseño de alimentos, para el caso de la panificación se han hecho estudios del uso de diferentes enzimas y su efecto sobre las propiedades de este alimento; en este sentido Jiang et al. (2010), evaluaron el efecto de la enzima xilanasa proveniente del Chaetomium sp., sobre el volumen de un pan elaborado al vapor; Selinheimo et al. (2006), evaluó el efecto de combinar las enzimas lacasa y xilanasa sobre las propiedades reológicas de una masa panaria. Así mismo, Caballero et al. (2007), estudiaron diferentes combinaciones de enzimas tales como la alfa-amilasa, xilanasa, proteasa, trasnglutaminasa, glucoxidasa, y lacasa para mejorar las propiedades reológicas de las masas panarias y el efecto de estas combinaciones sobre la vida útil del pan. Rosell y Singh (2004), mejoraron la calidad de un pan elaborado con harina de arroz con la adición de la enzima Glucosa Oxidasa. Otros estudios, como los de Madamwar et al. (2006), Stojceska et al. (2012), Schoenlechner et al. (2013) Vega et al. (2010) determinaron propiedades sensoriales físicas y fermentativas de panes formulados con diferentes tipos de enzimas, tales como la xilanasa amilasa, lipasa y gluco-oxidasa transglutaminasa y lacasa.

El objetivo del presente trabajo fue formular un pan a partir de tres enzimas -lacasa, xilanasa y lipasa-, evaluando diferentes propiedades físicas tales como el volumen del pan, peso, simetría, además de las características sensoriales del mismo (color de miga, textura, corteza, estructura aroma y sabor), siendo esta una formulación una alternativa hacia la generación de productos panificables saludables, es decir sin contenido de productos químicos. Las características físicas y sensoriales de los panes formulados, se compararon con la formulación de un pan con aditivos tradicionales como el ácido ascórbico y la azidocarbonamida.

\section{MATERIALES Y MÉTODOS}

\subsection{Materias primas}

Para la obtención de la harina utilizada en esta investigación, se molieron trigos cosechados en las localidades de Azul y 25 de Mayo, Provincia de Buenos Aires (Argentina). Las condiciones ambientales y geográficas de Azul y 25 de Mayo son: 137 msnm, con una temperatura media anual de $15^{\circ} \mathrm{Cy}$ por coordenadas 3646'39"S - 5951'48”0 y 58 msnm, con una temperatura media anual de $18^{\circ} \mathrm{C}$ y con coordenadas $35^{\circ} 25^{\prime} 41^{\prime \prime} \mathrm{S}-60^{\circ} 10^{\prime} 27^{\prime \prime} \mathrm{O}$, respectivamente. Los trigos se almacenaron durante 24 horas en silos de concreto y antes de la molienda se llevaron a una humedad del $16 \%$, la humedad inicial de los trigos fue del $11 \%$, después de la molienda el producto obtenido se mezcló para obtener una harina 000.

Para el caso de las enzimas estas fueron proveídas por diferentes empresas; la lacasa fue donada por Atime S.A., marca Muhlenchemie (Alphamalt 
PPO MC9901001), la síntesis de la enzima fue a partir del hongo Myceliophthera thermophila producida por fermentación sumergida de una cepa modificada genéticamente del Aspergillus orizae; la xilanasa se adquirió en Puratos Argentinos, con la denominación de 20ARG, el número de lote fue 25014; para el caso de la lipasa esta se adquirió en Granotec Argentina, bajo la denominación de Emulzime, el número de lote fue 2006127017. Finalmente, los agentes oxidantes, ácido ascórbico (ASC) y azidocarbonamida (ADA) fueron adquiridos en Epecuen S.A, con el número de lotes 7042605 y 70524001 , respectivamente.

Dentro de las materias primas para la elaboración del pan están: harina molida (ver numeral 1); levadura prensada o deshidratada: $3 \%$ o $1 \%$, respectivamente, en relación a la harina. Azúcar: 2,5 \% en relación con la harina. Sal: $1 \%$ en relación con la harina. Agua para análisis. Posteriormente se prepara una solución de azúcar y sal: para cada ensayo, 1 gramo de sal y 2,5 gramos de azúcar disueltos en 23,1 mililitros de agua. Para la suspensión de la levadura se pesan 3 gramos de levadura y se mezclan con 22,5 ml de agua, lo más homogéneamente posible.

Como equipos utilizados en la elaboración del pan, están una balanza de $\pm 0,01$ gramos, se utilizó una amasadora semirrápida la cual operaba a 90 v/min, además de una laminadora y moldeadora mecánica; para el moldeo del pan, se usaron moldes metálicos de chapa en acero de doble capa de $1.5 \mathrm{~mm}$ de espesor cuyas medidas son las siguientes: $9.5 \mathrm{~cm} \times 5.5 \mathrm{~cm}$ de base, $10.5 \mathrm{~cm} \times 7 \mathrm{~cm}$ de abertura superior y $5.5 \mathrm{~cm}$ de altura. Con respecto al proceso de fermentación, se usó una cámara de regulación automática de humedad ( $80 \pm 5 \%$ ) y temperatura $\left(30 \pm 1^{\circ} \mathrm{C}\right)$ y para el proceso de cocción se utilizó un horno eléctrico con resistencia en el piso.

\subsection{Elaboración del pan}

Para la elaboración del pan se tomó como base la Norma IRAM 15858-1 de diciembre de 1996. «Cereales; ensayo de panificacion experimental», método usado para ser usado en programas de mejoramiento de trigo. Antes de describir el proceso de elaboración del pan es preciso aclarar que se hicieron 7 formulaciones, las cuales se describen a continuación:

F1: harina 000;

F2: harina $000+\mathrm{ADA}+\mathrm{ASC}$;

F3: harina 000 + lacasa;

F4: harina $000+$ xilanasa + ADA + ASC;

F5: harina $000+$ lacasa + xilanasa;

F6: harina 000 + xilanasa + lipasa + ADA + ASC;

F7: harina 000 + lacasa + xilanasa + lipasa.

En cada una de las formulaciones, se utilizaron 15 ppm de ADA, 4 ppm de ASC, 200 ppm de lipasa, $100 \mathrm{ppm}$ de xilanasa y $50 \mathrm{ppm}$ de lacasa.

Las comparaciones realizadas en esta investigación fueron en función del papel que cada aditivo o enzima desempeña dentro de la formulación. Es decir F2 se comparó contra F3, debido a que los aditivos tanto químicos y enzimáticos cumplen un papel de carácter oxidativo en la reología de la masa panaria, para este caso los aditivos químicos el ADA y el ASC (Quaglia, 1991; Solito y Pavesi, 2003) y para la lacasa (Selinheimo et al., 2006); dado que los agentes oxidantes tienden a disminuir la elasticidad de la masa, se requiere una enzima o un agente que mejore esta propiedad, se hace entonces una nueva formulación con la adición de xilanasa, para mejorar la maquinabilidad de las masas (Williams y Pullen, 1998; Callejo, 2002) por ende se comparan las formulaciones F4 y F5; Finalmente, se decide agregar lipasa para mejorar la emulsión del sistema, dado una última comparación entre las formulaciones F6 y F7. F1 es un pan sin ningún tipo de aditivos.

Para la elaboración del pan se mezclan $100 \mathrm{~g}$ de harina con 25 mililitros tanto de la suspensión de levadura, solución de sal y azúcar, además de 16,5 $\mathrm{ml}$ de agua y se amasa durante 3.5 minutos. La temperatura final de la masa deberá ser de $27 \pm 1^{\circ} \mathrm{C}$. Posteriormente, se hace la pre-fermentación a $30 \pm$ $1^{\circ} \mathrm{C}$ y $80 \pm 5 \%$ por 85 minutos, durante esta parte del proceso es necesario hacer dos punchs que consisten en un amasado a mano en forma suave, para el desalojo del dióxido de carbono. El primer punchs 
se realiza 45 minutos de haber finalizado el amasado, el segundo punchs a los quince minutos del primero. Luego se arma el pan, colocando el bollo de masa para aplanarlo y alisarlo con un rodillo de madera hasta formar un disco de unos $8 \mathrm{~mm}$ de espesor, procediendo a formar la pieza de pan.

Para la fermentación final, se lleva el molde a la cámara de fermentación a $30^{\circ} \mathrm{C}$ y $80 \%$ por $75 \mathrm{mi}-$ nutos. Luego se hace la cocción, a $225^{\circ} \mathrm{C}$ con incorporación de vapor de agua mediante un inyector. El pan se cocina durante 15 minutos dentro del molde, se saca de este y se continúa la cocción hasta completar 30 minutos.

\subsection{Determinación de propiedades del pan}

Para la determinación de las propiedades del pan se hicieron con base en la Norma IRAM 15858-1 de diciembre de 1996 «Cereales; ensayo de panificación experimental», método para ser usado en programas de mejoramiento de trigo.

Volumen del pan: la medición del volumen del pan se hace una hora después de la cocción. Utilizando el aparato medidor de volumen, provisto de un recipiente que contiene el pan. La medición se realiza midiendo elvolumen de las semillas colza o nabo desplazados por el espacio ocupado por el pan. El volumen específico del pan se calculó como el cociente entre el volumen neto del pan y su peso.

Evaluación general: para evaluar la masa del pan, esta se debe dejar enfriar y se pesa. En el aspecto exterior del pan se evaluó la forma del pan, si es simétrico, el color y el brillo de la corteza. En el aspecto interior, se inicia a caracterizar dos horas después de sacado del horno, se corta y se evalúa la textura, estructura y color de miga.

La textura de la miga del pan se clasifica en una escala que comprende los siguientes límites superior e inferior: suave y elástica, áspera y rígida. Con respecto a la estructura, se verifica que la miga presente alvéolos homogéneos pequeños y de paredes finas a lo que le corresponde la evaluación óptima y, finalmente, el color debe ser blanco crema, el cual brinda la evaluación óptima. Cada uno de los pará- metros mencionados anteriormente, se evaluaron en función de los puntajes establecidos por la Norma IRAM 15858-1 de diciembre de 1996.

TABLA 1. VALORES DE ALGUNAS PROPIEDADES DEL PAN. Fuente: Norma IRAM 15858-1.

\begin{tabular}{c|c}
\hline Propiedad & Máximo Puntaje \\
\hline Volumen & 25 \\
\hline Corteza & 15 \\
\hline Textura & 15 \\
\hline Color de la miga & 15 \\
\hline Estructura & 10 \\
\hline Aroma & 10 \\
\hline Sabor & 10 \\
\hline
\end{tabular}

La evaluación de los panes se realizó, una hora después de la cocción. Fue evaluado por tres evaluadores entrenados del Molino Central Norte S.A., donde se efectuaron los ensayos correspondientes.

\subsection{Análisis sensorial}

Se realizó por medio de una prueba triangular, consistente en la presentación al evaluador de tres muestras, dos de las cuales son iguales. El degustador debe decidir cuál es la muestra diferente. La diferencia significativa de los resultados se evalúa tomando como referencia las tablas Bengtsson's con un nivel de significancia del $95 \%$.

Esta evaluación se llevó a cabo con dos paneles de evaluadores no entrenados, con un total de 38 jueces. El Panel 1se compuso de 31 evaluadores, y se dividió en dos grupos, de 15 y 16 evaluadores respectivamente. El Panel 2 fue compuesto por 7 evaluadores. Además, se realizaron tres preguntas adicionales a los evaluadores, donde se indagó acerca de la dificultad para identificar la muestra, de la intensidad con que cada evaluador percibió la muestra diferente, y por último se pidió que el evaluador expresara la muestra de preferencia.

La elaboración de las planillas de evaluación para el panel 1 se dividió en dos grupos; el grupo 1 de evaluadores se utilizó la siguiente notación: A: 
Pan con lacasa + xilanasa + lipasa; B y C: Pan con xilanasa + lipasa + ADA + ASC. Para el grupo 2 del panel 1 se usó la siguiente notación: A: pan con xilanasa + lipasa + ADA + ASC; B: pan con lacasa + xilanasa + lipasa. Para el panel 2 la notación fue la siguiente: A: pan con lacasa + xilanasa + lipasa; B y C: pan con xilanasa + lipasa + ADA+ASC. La identificación para cada tipo de muestra se realizó mediante la utilización de tablas de números aleatorios. Para cada repetición se usaron planillas diferentes. Las cuales se muestran en las Tablas 2, 3 y 4.

TABLA 2. PLANILLA DE CONTROL PARA EL GRUPO 1 DEL PANEL DE EVALUADORES NO. 1

\begin{tabular}{c|c|c|c|c|c|c}
\hline No. evaluador & \multicolumn{2}{|c|}{ Muestra 1 } & \multicolumn{2}{l|}{ Muestra 2 } & \multicolumn{2}{l}{ Muestra 3 } \\
\hline 1 & A & 785 & B & 137 & C & 377 \\
\hline 2 & A & 416 & C & 161 & B & 446 \\
\hline 3 & C & 490 & A & 714 & B & 141 \\
\hline 4 & C & 743 & B & 437 & A & 378 \\
\hline 5 & B & 358 & C & 529 & A & 537 \\
\hline 6 & B & 181 & A & 814 & C & 149 \\
\hline 7 & A & 430 & C & 309 & B & 979 \\
\hline 8 & B & 345 & A & 450 & C & 57 \\
\hline 9 & C & 527 & B & 373 & A & 228 \\
\hline 10 & B & 684 & A & 969 & C & 342 \\
\hline 11 & C & 565 & B & 537 & A & 591 \\
\hline 12 & A & 663 & C & 732 & B & 104 \\
\hline 13 & C & 590 & A & 778 & B & 429 \\
\hline 14 & C & 116 & B & 805 & A & 906 \\
\hline 15 & B & 965 & C & 769 & A & 485 \\
\hline
\end{tabular}

TABLA 3. PLANILLA DE CONTROL PARA EL GRUPO 2 DEL PANEL DE EVALUADORES NO. 1

\begin{tabular}{c|c|c|c|c|c|c|}
\hline No. Evaluador & \multicolumn{2}{|c|}{ Muestra 1 } & \multicolumn{2}{c|}{ Muestra 2 } & \multicolumn{2}{c}{ Muestra 3 } \\
\hline 16 & B & 908 & A & 445 & C & 467 \\
\hline 17 & A & 664 & C & 14 & B & 511 \\
\hline 18 & A & 328 & B & 642 & C & 567 \\
\hline 19 & C & 628 & B & 114 & A & 140 \\
\hline
\end{tabular}

\begin{tabular}{c|c|c|c|c|c|c}
20 & B & 798 & A & 319 & C & 240 \\
\hline 21 & A & 876 & B & 660 & C & 537 \\
\hline 22 & A & 135 & C & 410 & B & 831 \\
\hline 23 & C & 49 & A & 59 & B & 37 \\
\hline 24 & C & 15 & B & 8 & A & 72 \\
\hline 25 & B & 73 & C & 29 & A & 91 \\
\hline 26 & B & 64 & A & 55 & C & 37 \\
\hline 27 & A & 10 & C & 93 & B & 86 \\
\hline 28 & B & 51 & A & 18 & C & 20 \\
\hline 29 & C & 96 & B & 73 & A & 28 \\
\hline 30 & B & 69 & A & 99 & C & 58 \\
\hline 31 & C & 49 & B & 31 & A & 94 \\
\hline
\end{tabular}

TABLA 4. PLANILLA DE CONTROL PARA EL PANEL DE EVALUADORES NO. 2

\begin{tabular}{c|c|c|c|c|c|c|}
\hline No. evaluador & \multicolumn{2}{|c|}{ Muestra 1 } & \multicolumn{2}{c|}{ Muestra 2 } & \multicolumn{2}{c}{ Muestra 3 } \\
\hline 32 & A & 79 & A & 83 & B & 19 \\
\hline 33 & A & 62 & B & 81 & A & 14 \\
\hline 34 & B & 32 & A & 86 & A & 42 \\
\hline 35 & A & 96 & B & 77 & C & 53 \\
\hline 36 & B & 57 & C & 84 & A & 75 \\
\hline 37 & C & 69 & A & 29 & B & 91 \\
\hline 38 & B & 71 & A & 23 & A & 12 \\
\hline
\end{tabular}

\subsection{Análisis estadístico}

Se realizó un diseño de experimentos libre al azar, tomado como único factor la formulación del pan, el cual tenía 7 niveles, como variables respuesta se tomaron el volumen del pan $\mathrm{cm}^{3}$, el volumen especifico $\mathrm{cm}^{3} / \mathrm{g}$ y el color del pan. Los datos se analizaron por medio de una tabla anova, con un nivel de significancia del $95 \%$, esto para establecer si existían o no diferencias significativas entre los grupos. Para establecer entre que grupos existían diferencias se realizó un análisis tipo LSD. El software usado fue el STATGRAPHICS Centurión XVI.I.

\section{RESULTADOS Y DISCUSIÓN}

A continuación se muestran, en la Tabla 5, los valores obtenidos para cada uno de los ítems. 
Comparando los datos obtenidos en esta investigación, respecto a los encontrados en la bibliografía, Chen et al. (2014), hallaron valores para el volumen especifico entre 2,5 a $3,75 \mathrm{~cm}^{3} / \mathrm{g}$, para un pan elaborado con Bifidobacterium lactis Bb12, estos valores menores a los reportados en esta investigación, sin embargo pude deberse a que el tiempo de horneado en el trabajo de Chen et al. (2014), fue de 12 minutos y el de esta investigación fue de 15 minutos y el tiempo de horneado influye en esta variable respuesta, ya que a mayor tiempo de horneo mayor será el volumen específico. Jiang et al. (2010) y Caballero et al. (2007) dan valores para el volumen específico entre $2,04-2,54 \mathrm{~cm}^{3} / \mathrm{g}$ y $3,22-4,17 \mathrm{~cm}^{3} / \mathrm{g}$, estos valores para panes formulados con xilanasa y otras enzimas como transglutaminasa, glucosoxidasa y lacasa, respectivamente. Autores como (Rosell y Singh, 2004; Madamwar et al., 2006; Stojceska et al., 2012; y Schoenlechner et al., 2013) encontraron valores que oscilan entre $1,6-3,73 \mathrm{~cm}^{3} / \mathrm{g}$, para el volumen específico, todo en función de la formulación del pan. Para panes libres de gluten Gamonpilas et al. (2014) reporta valores 1,85 a $2,84 \mathrm{~cm}^{3} / g$.

Para el volumen del pan, Noor et al. (2013), Korus et al. (2010) y Shittu et al. (2007) obtuvieron rangos de valores de 544-804 $\mathrm{cm}^{3}, 600-800 \mathrm{~cm}^{3} \mathrm{y}$ $440 \mathrm{~cm}^{3}$ a $920 \mathrm{~cm}^{3}$, para panes formulados a partir de banano y trigo con diferentes tipos de maltodextrinas y concentraciones de las mismas y formulaciones hechas con yuca y harina de trigo.

TABLA 5. VALORES PROPIEDADES FÍSICAS Y SENSORIALES PARA CADA FORMULACIÓN

\begin{tabular}{|c|c|c|c|c|c|c|c|}
\hline CARACTERÍSTICASMASA & $\boldsymbol{F 1}$ & $\boldsymbol{F 2}$ & $F 3$ & $F 4$ & F5 & F6 & F7 \\
\hline Peso de la masa & 819 & 827 & 829 & 816 & 836 & 834 & 824 \\
\hline Peso del Pan & 691,7 & 703,74 & 707,46 & 714,76 & 701,04 & 690,22 & 676,52 \\
\hline Volumen del pan $\mathrm{cm}^{3}$ & 560 & 476 & 480 & 676 & 652 & 792 & 784 \\
\hline Rendimiento por kg. & $38,34 \%$ & $40,75 \%$ & $41,49 \%$ & $42,95 \%$ & $40,21 \%$ & $38,04 \%$ & $35,30 \%$ \\
\hline Perdida de Agua en el Horno & $15,54 \%$ & $14,90 \%$ & $14,66 \%$ & $12,41 \%$ & $16,14 \%$ & $17,24 \%$ & $17,90 \%$ \\
\hline Perdida de Agua \% & $38,29 \%$ & $36,79 \%$ & $36,28 \%$ & $30,22 \%$ & $40,29 \%$ & $42,92 \%$ & $44,35 \%$ \\
\hline \multicolumn{8}{|l|}{ CARACTERÍSTICAS EXTERNAS } \\
\hline Volumen & 12,15 & 10,14 & 10,17 & 14,19 & 13,95 & 16,08 & 15,69 \\
\hline Simetría & $A^{*}$ & $S^{*}$ & $S$ & $S$ & $S$ & $\mathrm{~S}$ & $\mathrm{~S}$ \\
\hline Color de la corteza & 8 & 8 & 9 & 12 & 12 & 10 & 13 \\
\hline \multicolumn{8}{|l|}{ CARACTERÍSTICAS INTERNAS } \\
\hline Textura & 10 & 7 & 10 & 12 & 11 & 10 & 12 \\
\hline Estructura & 5 & 5 & 8 & 10 & 9 & 11 & 10 \\
\hline Sabor & 10 & 10 & 10 & 10 & 8 & 9 & 8 \\
\hline Aroma & 5 & 10 & 7 & 10 & 10 & 10 & 10 \\
\hline Color & 5 & 8 & 8 & 10 & 8 & 11 & 10 \\
\hline Volumen específico $\mathrm{cm}^{3} / \mathrm{g}$ & 4,05 & 3,38 & 3,39 & 4,73 & 4,65 & 5,36 & 5,23 \\
\hline Peso específico $\mathrm{g} / \mathrm{cm}^{3}$ & 0,25 & 0,3 & 0,29 & 0,21 & 0,22 & 0,19 & 0,19 \\
\hline PUNTAJE FINAL & 60,15 & 66,14 & 70,17 & 88,19 & 80,95 & 77,08 & 78,69 \\
\hline
\end{tabular}


Madamwar et al. (2006) determinó el volumen de un pan formulado con xilanasa y salvado de trigo, obteniendo un valor de $460 \mathrm{~cm}^{3}$ con respecto al pan control que fue de $300 \mathrm{~cm}^{3}$. Este resultado está en concordancia con los resultados reportados en esta investigación ya que con el agregado de la enzima xilanasa, es decir, los tratamientos F4 y F5 (ver Tabla 5), el volumen del pan se incrementó debido a que la xilanasa mejora la extensibilidad de la masa y por ende esta puede retener más gas y lo que se traduce en un mayor volumen del pan. Finalmente, Mishra et al. (2012) reporta volúmenes de 139-165 $\mathrm{cm}^{3}$ para panes formulados con harina de mijo.

Los análisis de varianza muestra que para la variable respuesta volumen especifico, existieron diferencias significativas $(\mathrm{P}<0,05)$, para las diferentes formulaciones, excepto entre la F2 y F3. El mismo comportamiento arrojó la variable respuesta volumen de pan, donde F2 y F3 no mostraron diferencias significativas, sin embargo las demás formulaciones el valor $\mathrm{p}$ fue $<0,05$. Con respecto al color, la tabla ANOVA mostró un $\mathrm{p}<0,05$; y el análisis de mínimas diferencias significativas encontró homogeneidad entre las F2, F3 y F5. Estos resultados se pueden ver en la Figura 1 donde se muestran las gráficas de LSD para estas variables respuestas.
Con respecto a la formulación F1, las formulaciones F2 y F3 disminuyeron el volumen del pan un $14,5 \%$ aproximadamente; esto probablemente es debido al aumento de tenacidad y en el caso de los oxidantes químicos también al aumento de elasticidad. También se aprecia una disminución del volumen específico. Virtanen et al. (1999) reportaron que la lacasa proveniente de Myceliophthora thermophila producía un incremento en el volumen del pan, lo cual es contrario a los resultados obtenidos para F3. En dicho trabajo se reporta que el $\mathrm{pH}$ de trabajo es 7,0; las condiciones del presente ensayo fueron de $\mathrm{pH}$,9 y es probable que esto haya influido en las diferencias reportadas.

Existe una diferencia desde el punto de vista de la calidad de la miga: el tratamiento con lacasa formó una miga más húmeda y esponjosa, con alvéolos pequeños. En cambio, con la adición de ADA-ASC, la miga formada carece de elasticidad, los alvéolos son muy irregulares y algunos de gran tamaño, en concordancia con lo descrito por Ribolta y León (1999); esto último se puede observar en fotografía del corte de ambas panificaciones (Figura 2a y 2b). En la misma se aprecia también que el uso de oxidantes químicos generó una corteza más gruesa comparándola con la panificación de la

Figura 1. Análisis LSD p 0.05 para color, volumen y volumen específico de los panes.
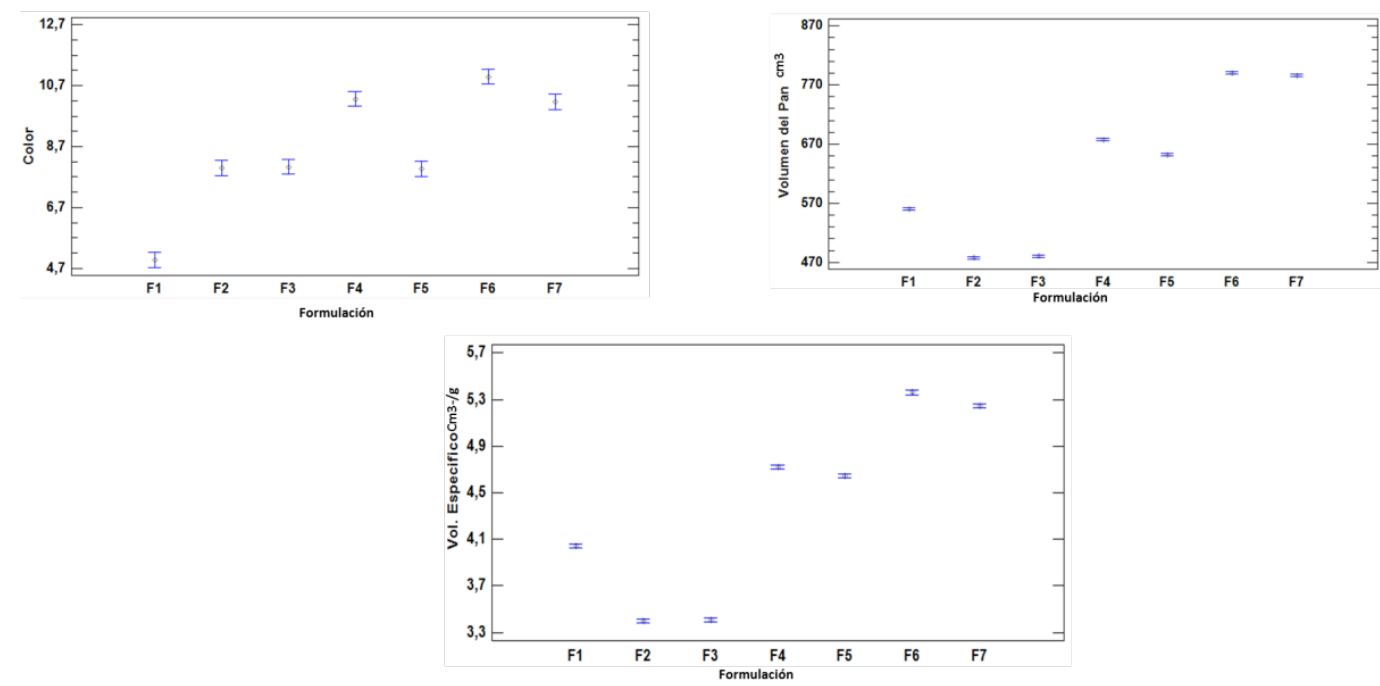
harina aditivada con lacasa. En la Figura 2 se puede observar la diferencia entre el tamaño de los alvéolos y las características de la miga, lo que puede indicar que el pan formulado con la enzima lacasa es mejor que el de los aditivos químicos, lo cual se evidencia con el puntaje asignado por los jueces.

Para el caso de las formulaciones F4 y F5 a la adición de la xilanasa conjuntamente con los oxidantes generó una masa más extensible favoreciendo el aumento del volumen y mejorando la textura de la miga con alvéolos más uniformes. La Figura 3 mues- tra las fotografías de las formulaciones 4 y 5 , donde se ilustra lo mencionado anteriormente. Cabe destacar que las diferencias en el puntaje final se debieron fundamentalmente a diferencias en la calidad de la miga. Sin embargo, estas diferencias se pueden deber a una inhibición del efecto que existe entre las dos enzimas, la cual es reportada por Selinheimo (2006). Sin embargo y de acuerdo a los puntajes asignados por los jueces, los panes obtenidos con esa nueva formulación tienen una puntuación por encima de 80 puntos.

Figura 2. Fotografías de los panes obtenidos con la formulación de ADA-ASC F2 (2a) y la formulación con lacasa F3 (2b).

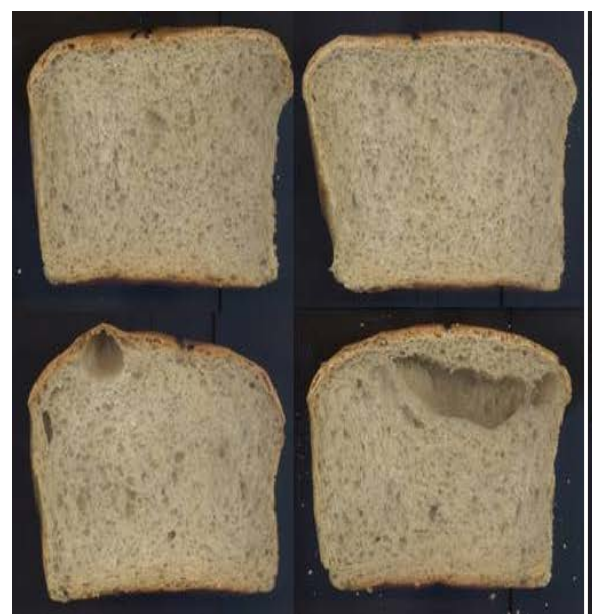

$2 a$

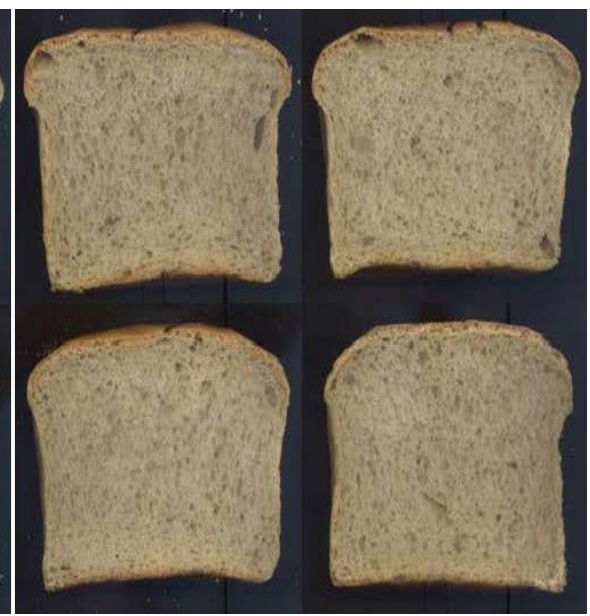

$2 b$

Figura 3. Fotografías de los panes obtenidos con la formulación de F4 (3a) y la formulación F5 (3b).

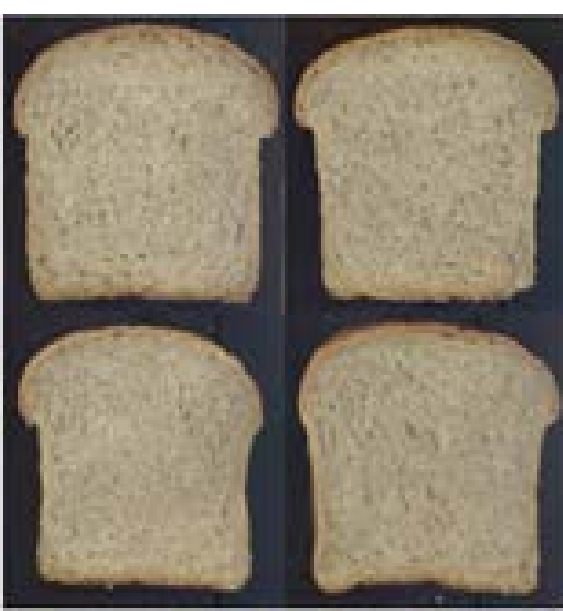

$3 a$

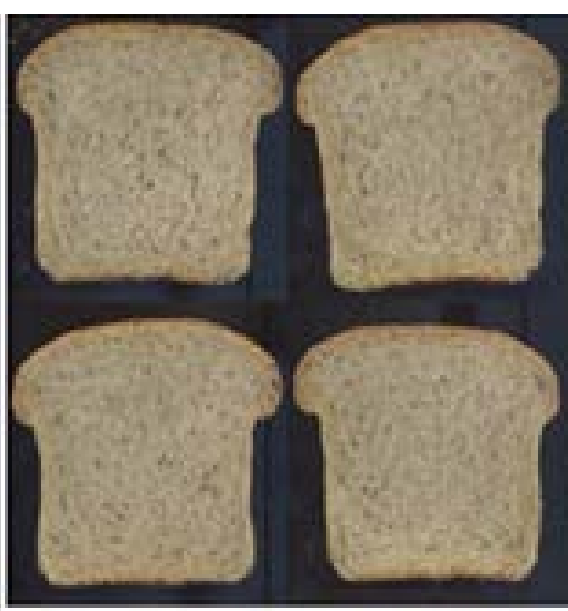

$3 b$ 
Figura 4. Fotografías de los panes obtenidos con la formulación F6 (4a) y la formulación con F7 (4b).

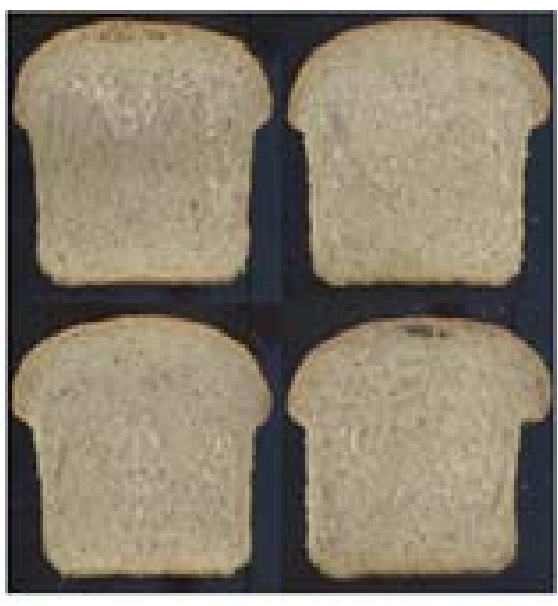

$4 a$

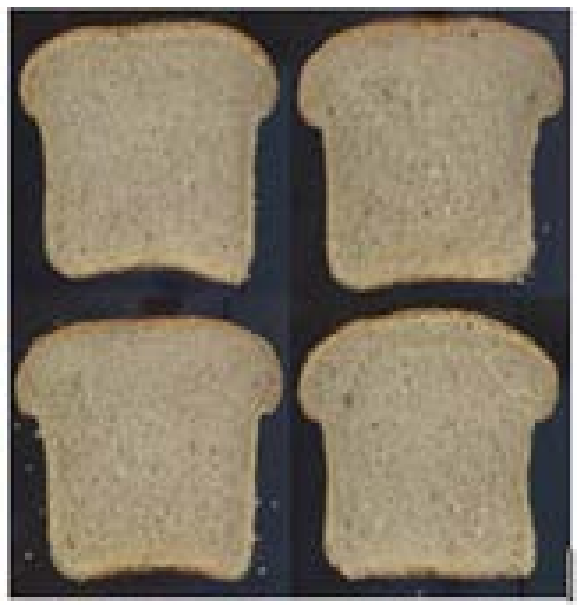

$4 \mathrm{~b}$
El agregado de lipasas para las formulaciones F6 y F7 produjo una suavidad en la miga una relajación de la masa, posibilitando la formación de estructura alveolar más uniforme y asimismo un mayor volumen, comparando con los resultados obtenidos en las formulaciones F4 y F5. Es importante destacar que la textura lograda en la formulación F7 (lacasa-xilanasa-lipasa) fue más esponjosa y elástica, la misma obtuvo una calificación de 12 de acuerdo a especificaciones de la Norma IRAM 15858 es la máxima calificación, mientras que para el formulación 6 (ADA-ASC-xilanasa-lipasa) el puntaje fue de 10.

Con respecto al color de la miga, en la F7 se observó una miga algo más oscura que en la F6, esto puede deberse a que los oxidantes químicos, en general, producen una masa más blanca. Salvo las dos consideraciones anteriores, no se encontró gran diferencia entre las dos últimas determinaciones, por lo tanto se puede concluir que el F7 hace del pan un excelente producto.

En la Figura 4 se puede observar la calidad de los panes que corresponden a las F6 y F7, donde se puede observar una miga uniforme, color y corteza, estas fotografías corresponden a los puntajes asignados por los jueces.

\subsection{Análisis sensorial}

Los resultados del análisis sensorial realizado por los dos paneles de evaluadores presentan en la Tabla 6. Se decidió realizar un análisis de este tipo únicamente para contrastar las formulaciones F6 y F7, de acuerdo a los resultados obtenidos anteriormente.

Del total de 38 evaluadores, los jueces número 2, 17,20 y 28 no seleccionaron muestra diferente, para corregir el error la evaluación se hizo sobre 34 evaluadores. Con los resultados obtenidos no se encontraron diferencias significativas entre el pan formulado con lacasa-xilanasa-lipasa y el formulado con xilanasalipasa-ADA-ASC. No existieron diferencias significativas porque para un nivel del $5 \%$ y para 34 jueces, era necesario como mínimo que 17 jueces identificaran la muestra diferente y en la degustación realizada en la presente investigación solamente 11 jueces identificaron la muestra diferente. Los jueces que identificaron la muestra diferente fueron: 1, 6, 13, 14, 16, 18, 22, 25, 32,36 y 38. En cuanto a la preferencia según el tipo de formulación de pan, el 39 \% de los jueces prefirió el pan formulado con lacasa, el 43 \% prefirió la formulación sin lacasa y el porcentaje restante no emitió preferencia. En sí, las preferencias fueron muy similares, como puede apreciarse en el gráfico presentado en la Figura $\mathbf{5}$, lo cual hace aceptable al pan formulado con lacasa. 


\section{TABLA 6. RESULTADOS DE LA PRUEBA DE ANÁLISIS SENSORIAL PARA LAS FORMULACIONES F6 Y F7}

\begin{tabular}{|c|c|c|c|c|c|c|c|c|c|c|}
\hline No. Evaluador & \multicolumn{2}{|c|}{ Muestra 1} & \multicolumn{2}{|c|}{ Muestra 2} & \multicolumn{2}{|c|}{ Muestra 3} & Muestra Dif. & Inten.Dife. & Ident. Dife. & M. Prefer. \\
\hline \multicolumn{11}{|c|}{ A: Pan con Lacasa, B y C: Pan con ADA y ASC } \\
\hline 1 & A & 785 & B & 137 & C & 377 & 785 & I & $\mathrm{F}$ & 785 \\
\hline 2 & A & 416 & C & 161 & $B$ & 446 & & $\mathrm{D}$ & $\mathrm{Cl}$ & 416 \\
\hline 3 & C & 490 & A & 714 & $B$ & 141 & 141 & MD & Md & 141 \\
\hline 4 & C & 743 & B & 437 & A & 378 & 437 & MD & $\mathrm{Cl}$ & \\
\hline 5 & $B$ & 358 & C & 529 & A & 537 & 358 & $M D$ & $\mathrm{Di}$ & 537 \\
\hline 6 & $\mathrm{~B}$ & 181 & A & 814 & C & 149 & 814 & $\mathrm{D}$ & $\mathrm{Di}$ & 181 \\
\hline 7 & A & 430 & C & 309 & B & 979 & 309 & $\mathrm{MD}$ & $\mathrm{F}$ & 309 \\
\hline 8 & $B$ & 345 & A & 450 & C & 57 & 345 & $\mathrm{D}$ & MF & 450 \\
\hline 9 & C & 527 & B & 373 & A & 228 & 527 & MD & $\mathrm{Cl}$ & \\
\hline 10 & $\mathrm{~B}$ & 684 & A & 969 & $\mathrm{C}$ & 342 & 342 & $\mathrm{D}$ & $\mathrm{Di}$ & 684 \\
\hline 11 & $C$ & 565 & $B$ & 537 & A & 591 & 537 & $\mathrm{D}$ & $\mathrm{Di}$ & 565 \\
\hline 12 & A & 663 & C & 732 & B & 104 & 732 & $\mathrm{D}$ & $\mathrm{Di}$ & 104 \\
\hline 13 & C & 590 & A & 778 & $\mathrm{~B}$ & 429 & 778 & $\mathrm{MD}$ & Md & 778 \\
\hline 14 & C & 116 & B & 805 & A & 906 & 906 & $\mathrm{D}$ & $\mathrm{Di}$ & \\
\hline 15 & B & 965 & C & 769 & A & 485 & 965 & $\mathrm{MD}$ & $\mathrm{Cl}$ & 965 \\
\hline \multicolumn{11}{|c|}{ A:Pan con ADA y ASC; B y C: Pan con Lacasa } \\
\hline 16 & B & 908 & A & 445 & C & 467 & 445 & M & $\mathrm{F}$ & \\
\hline 17 & A & 664 & C & 14 & $B$ & 511 & & $\mathrm{D}$ & $\mathrm{Di}$ & 664 \\
\hline 18 & A & 328 & B & 642 & C & 567 & 328 & C & $\mathrm{F}$ & 642 \\
\hline 19 & $C$ & 628 & $B$ & 114 & $\mathrm{~A}$ & 140 & 628 & $\mathrm{D}$ & $\mathrm{F}$ & 628 \\
\hline 20 & B & 798 & A & 319 & C & 240 & & C & $\mathrm{F}$ & 240 \\
\hline 21 & A & 876 & B & 660 & C & 537 & 660 & $\mathrm{D}$ & $\mathrm{Di}$ & 876 \\
\hline 22 & $\mathrm{~A}$ & 135 & $C$ & 410 & $B$ & 831 & 135 & $\mathrm{D}$ & $\mathrm{F}$ & 135 \\
\hline 23 & C & 49 & A & 59 & B & 37 & 37 & D & $\mathrm{F}$ & \\
\hline 24 & C & 15 & B & 8 & A & 72 & 8 & D & $\mathrm{Md}$ & \\
\hline 25 & $B$ & 73 & $C$ & 29 & A & 91 & 91 & $M$ & $\mathrm{Di}$ & 73 \\
\hline 26 & B & 64 & A & 55 & C & 37 & 37 & C & $\mathrm{F}$ & 64 \\
\hline 27 & $\mathrm{~A}$ & 10 & $C$ & 93 & $\mathrm{~B}$ & 86 & 93 & $M$ & $\mathrm{~F}$ & 93 \\
\hline 28 & $B$ & 51 & A & 18 & $C$ & 20 & & $C$ & $\mathrm{MF}$ & 20 \\
\hline 29 & C & 96 & B & 73 & A & 28 & 96 & $\mathrm{MD}$ & $\mathrm{Md}$ & \\
\hline 30 & $B$ & 69 & $\mathrm{~A}$ & 99 & $C$ & 58 & 69 & $M$ & $\mathrm{~F}$ & 69 \\
\hline 31 & $C$ & 49 & B & 31 & A & 94 & 49 & $M$ & $\mathrm{Md}$ & 31 \\
\hline \multicolumn{11}{|c|}{ A: Pan con Lacasa, B y C: Pan con ADA y ASC } \\
\hline 32 & A & 79 & A & 83 & $\mathrm{~B}$ & 19 & 19 & $\mathrm{D}$ & $\mathrm{F}$ & 79 \\
\hline 33 & A & 62 & B & 81 & A & 14 & 14 & $M$ & $\mathrm{~F}$ & 81 \\
\hline 34 & B & 32 & A & 86 & A & 42 & 42 & M & $\mathrm{F}$ & 32 \\
\hline 35 & A & 96 & B & 77 & C & 53 & 77 & D & $\mathrm{F}$ & 77 \\
\hline 36 & B & 57 & C & 84 & A & 75 & 75 & $M$ & $\mathrm{Di}$ & 57 \\
\hline 37 & C & 69 & A & 29 & B & 91 & 91 & $\mathrm{M}$ & $\mathrm{F}$ & 91 \\
\hline 38 & B & 71 & A & 23 & A & 12 & 71 & $D$ & $\mathrm{Di}$ & 71 \\
\hline
\end{tabular}

* La casilla denominada como muestra diferente, se refiere al número de muestra seleccionada por el evaluador como distinta. D:Débil, I: Intensa; MD: Muy Débil; M: Moderada; C: Clara. F: Fácil; Di: Difícil; Md: Muy Difícil; Cl: Casi Imposible. 
Con respecto a la facilidad de identificación de la muestra, un $42 \%$ de los jueces aseguró que fue fácil identificar la muestra diferente, en tanto que un 29 \% contestó que la identificación de la muestra fue difícil, un $13 \%$ aseguró que fue muy difícil, el $11 \%$ entendió que identificar la muestra diferente era casi imposible y, por último, al $5 \%$ de los jueces les fue muy fácil encontrar la degustación diferente, como se muestra gráficamente en la Figura 6.

Con respecto a la intensidad con que los jueces percibieron la muestra diferente, el $41 \%$ aseguró que fue débil dicha percepción, un $29 \%$ percibió como moderada la intensidad, el $16 \%$ de los jueces respondió que la intensidad muy débil, y por último el $11 \%$ y $3 \%$ percibió como clara e intensa la percepción de la diferencia (Figura 7).

La conclusión del análisis sensorial es que el mismo no reportó diferencias significativas entre la formulación del pan realizado con lacasa-xilanasa-lipasa y el pan formulado con xilanasa-ADA-ASC-lipasa.

Figura 5. Porcentaje de preferencia del pan según la formulación.

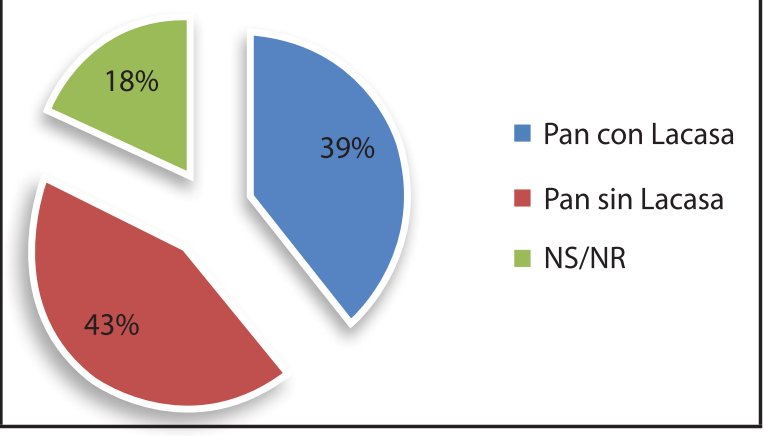

Figura 6. Porcentaje el grado de dificultad con que los evaluadores identificaron la muestra diferente.

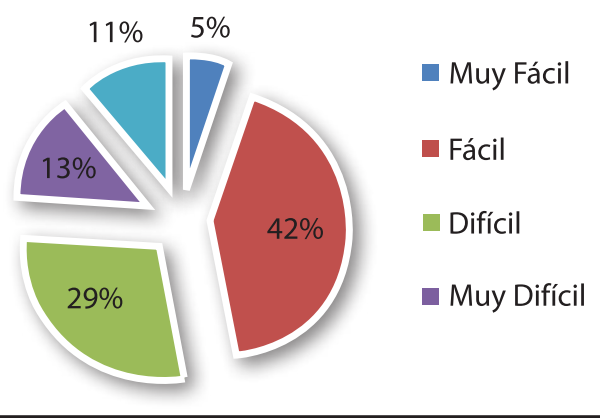

Figura 7. Grado de intensidad con que los evaluadores percibieron la muestra diferente.

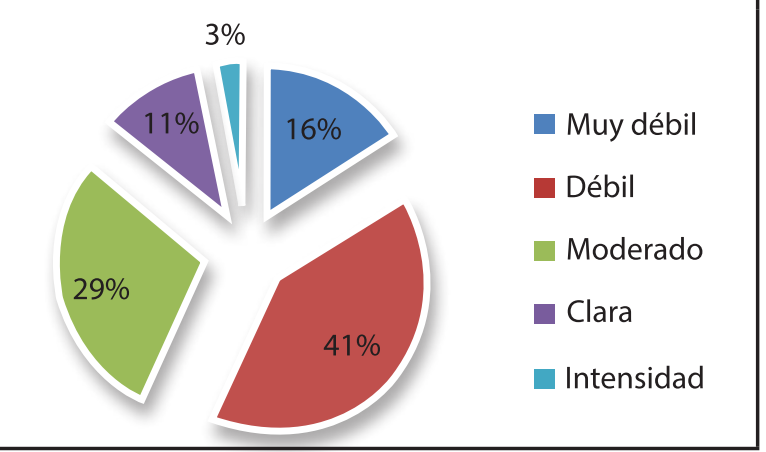

\section{CONCLUSIONES}

La adición de una mezcla de enzimas lacasaxilanasa-lipasa a una masa compuesta por harina de trigo, levadura, sal, azúcar y agua, genera un buen «oven spring», obteniendo un aumento de volumen en el horno. Además, que las características internas del pan tales como la miga, tienen una distribución alveolar homogénea y con textura esponjosa. Dadas estas características se realizó un análisis sensorial del producto obtenido, el cual demostró que la mezcla propuesta permite obtener un producto de buena calidad y aceptabilidad por el consumidor. Se concluye, entonces, que con una estrategia de combinación de enzimas puede lograrse una harina para panificación de calidad comparable a la obtenida con los aditivos químicos y enzimas utilizados en la actualidad. Los resultados obtenidos hasta el momento son promisorios en la búsqueda de sustituyentes enzimáticos a los aditivos oxidantes químicos en panificación. Se puede concluir entonces que la combinación de las tres enzimas utilizadas (laccasa, xilanasa y lipasa) dio un lugar a un panificado de buenas características.

\section{AGRADECIMIENTOS}

Los autores agradecen todo el apoyo prestado por la empresa Molinos Central del Norte (Burzaco, Buenos Aires-Argentina), en cuanto a equipos de laboratorio, jueces entrenados y logística en general, además de los estudiantes de Licenciatura 
de Alimentos, de la Universidad Nacional de Lanús. Además, al programa de sostenibilidad de Grupos de Investigación 2014-2016 U. de A.

\section{REFERENCIAS}

\section{http://www.fenalco.com.co/contenido/1476}

Abdelrahman, R.; Ahmed, I. y Mohammed, B. (2012). Dough Rheology and Bread Quality of Wheat-Chickpea Flour Blends. Industrial Crops and Products, 36(1), March, p.p. 196-202.

Arasaratnam, V.; et al. (2010). Optimization of Bread Preparation from Wheat Flour and Malted Rice Flour. Rice Science, 17(1), March, p.p. 51-59.

Arendt, E.; Hüttner, K. y Dal Bello, F. (2010). Rheological Properties and Bread Making Performance of Commercial Whole Grain Oat Flours, Journal of Cereal Science. 52(1), July, p.p. 65-71.

Baiano, A. et al. (2009). Physical and Mechanical Properties of Bread Loaves Produced by Incorporation of Two Types of Toasted Durum Wheat Flour. Journal of Food Engineering, 95(1), November, p.p.199-207.

Batifouliera, F. et al. (2005). Effect of Different Breadmaking Methods on Thiamine, Riboflavin and Pyridoxine Contents of Wheat Bread. Journal of Cereal Science, 42(1), July, p.p.101-108.

Bovell-Benjamin, A. et al. (2008). Comparison of chemical, physical, micro-structural, and microbial properties of breads supplemented with sweetpotato flour and high-gluten dough enhancers. Lebensmittel-Wissenschaft und-Technologie, 41(5), June, p.p. 803-815.

Caballero, P.; Gómez, M. y Rosell, C. (2007). Improvement of Dough Rheology, Bread Quality and Bread ShelfLife by Enzymes Combination. Journal of Food Engineering, 81(1), July, p.p. 42-53.

Chen, X. et al. (2014). A Study on Bifidobacterium Lactis Bb12 Viability in Bread During Baking. Journal of Food Engineering, 122, February, p.p. 33-37.

Demigne, C. et al. (2006). Variability of B vitamin concentrations in wheat grain, milling fractions and bread products. European Journal Agronomy, 25(2), August, p.p.163-169.

Gamonpilas, C. et al. (2014). Influence of Pregelatinised Tapioca Starch and Transglutaminase on Dough Rheology and Quality of Gluten-Free Jasmine Rice Breads. Food Hydrocolloids, 36, May, p.p 143-150.
Haros, M. et al. (2013). Effect of Whole Amaranth Flour on Bread Properties and Nutritive Value. LWT - Food Science and Technology, 50(3), March, p.p. 679-685.

Jiang, Z.; Cong, Q.; Yan, Q.; Kumar, N.; Du, X. (2010). Characterisation of a Thermostable Xylanase From Chaetomium sp. and its Application in Chinese Steamed Bread. Food Chemistry, 120, May, p.p. 457-462.

Kim, Y.; Shin, D. y Kim, W. (2013). Physicochemical and Sensory Properties of Soy Bread Made with Germinated, Steamed, and Roasted Soy Flour. Food Chemistry, 141(1), November, p.p. 517-523.

Korus, J. et al. (2010). The Effects of Maltodextrins on Gluten-Free Dough and Quality of Bread. Journal of Food Engineering, 96(2), January, p.p. 258-265.

Korus, J. et al. (2013). Supplementation of Gluten-Free Bread with Non-Gluten Proteins. Effect on Dough Rheological Properties and Bread Characteristic. Food Hydrocolloids, 32(2), August, p.p. 213-220.

Laureati, M.; Giussani, B. y Pagliarini, E. (2012). Sensory and Henic Perception of Gluten-Free Bread: Comparison Between Celiac and Non-Celiac Subjects. Food Research International, 46(1), April, p.p. 326333.

Madamwar, D.; Shah, A. y Shah, R. (2006). Improvement of the Quality of Whole Wheat Bread by Supplementation of Xylanase from Aspergillus Foetidus. Bioresource Technology, 97(16), November, p.p. 2047-2053.

Mandala, I. (2005). Physical Properties of Fresh and Frozen Stored, Microwave-Reheated Breads, Containing Hydrocolloids. Journal of Food Engineering, 66(3), February, p.p. 291-300.

Mahmoud, R. et al. (2013). Formulations and Quality Characterization of Gluten-Free Egyptian Balady Flat Bread. Annals of Agricultural Science, 58(1), June, p.p. 19-25.

Mihhalevski, A. et al. (2013). Stability of B-Complex Vitamins and Dietary Fiber During Rye Sourdough Bread Production. Journal of Cereal Science, 57(1), January, p.p. 30-38.

Mishra, A.; Singh, K. y Mishra, H. (2012). Fuzzy Analysis of Sensory Attributes of Bread Prepared from MilletBased Composite Flours. LWT - Food Science and Technology, 48(2), October, p.p. 276-282.

Morais, E. et al. (2014). Prebiotic Gluten-Free Bread: Sensory Profiling and Drivers of Liking. LWT - Food Science and Technology, 55(1), January, p.p. 248-254. 
Noor, A.; Lee-Hoon, H. y Baharin, A. (2013). PhysicoChemical Characteristics and Sensory Evaluation of Wheat Bread Partially Substituted with Banana (Musa acuminata X balbisiana cv. Awak) PseudoStem Flour. Food Chemistry, 139(1-4), August, p.p. 532-539.

Perez, G. et al. (2012). Incorporation of Several Additives into Gluten Free Breads: Effect on dough properties and bread quality. Journal of Food Engineering, 111(4), August, p.p. 590-597.

Quaglia, G. (1991). Ciencia y Tecnología de la Panificación. Editorial Acriba: Zaragoza, España. 485 p.

Ribotta, P.; Morcillo, M. y León, A. (1999). Efecto de distintos oxidantes sobre la calidad de panes elaborados por el método tradicional Argentino. Agriscientia, 16 , Septiembre, p.p. 3-10.

Rosell, C. et al. (2012). Viability of Some Probiotic Coatings in Bread and its Effect on the Crust Mechanical Properties. Food Hydrocolloids, 29(1), October, p.p. 166-174.

Rosell, C. y Singh, H. (2004). Improvement of the Bread Making Quality of Rice Flour by Glucose Oxidase. Food Research International, 37(1), January, p.p.7581.

Selinheimo, E. et al. (2006). Effects of Laccase, Xylanase and Their Combination on the Rheological Properties of Wheat Doughs. Journal of Cereal Science, 43(2), March, p.p. 152-159.

Shittu, T.; Raji, A. y Sanni, L. (2007). Bread From Composite Cassava-Wheat Flour: I. Effect of Baking Time and Temperature on Some Physical Properties of Bread Loaf. Food Research International, 40(2), March, p.p. 280-290.

Schoenlechner, et al. (2013). Optimization of Bread Quality Produced From Wheat and Proso Millet (Panicum miliaceum L.) by Adding Emulsifiers, Transglutaminase and Xylanase. LWT - Food Science and Technology, 51(1), April, p.p. 361-366.

Solito, A. y Pavesi, R. (2003). Tecnología, Camino a la salud: Reemplacemos el Bromato de Potasio. Argentina: Cámara Argentina de Concesionarios de Servicios de Comedores y Refrigerios. Universidad Nacional de Lanus. 50 p.

Stojceska, V. et al. (2012). Improving the Quality of Nutrient-Rich Teff (Eragrostis tef) Breads by Combination of Enzymes in Straight Dough and Sourdough
Breadmaking. Journal of Cereal Science, 55(1), January, p.p. 22-30.

Williams, T. y Pullen, G. (1998). Ingredientes Funcionales. En: Cauvain, S.; Young, L. Fabricación de Pan. Editorial Acribia: Zaragoza, España. p.p. 51-92.

Van der Goot-Atze, J. et al. (2011). Preparation of GlutenFree Bread Using a Meso-Structured Whey Protein Particle System. Journal of Cereal Science, 53(3), May, p.p. (355-361).

Virtaren, S. et al. (1999). Modification of Wheat Gluten with Oxidative Enzymes. Proceedings of the 2nd. In: European Symposium on Exymen in Grain Processing.; T. Simoinen- M. Tenkanen (Eds.).; Helsinki, Finlandia, 8-10 Dec.

Vodovotz, Y. y Alessia, L. (2008). Physical Properties and Water State Changes During Storage in Soy Bread with and Without Almond. Food Chemistry, 110(3), October, p.p. 554-561.

Zhou, W.; Rong, W. y Mia, I. (2007). Comparison Study of the Effect of Green Tea Extract (GTE) on the Quality of Bread by Instrumental Analysis and Sensory Evaluation. Food Research International, 40(4), May, p.p. 470-479.

\section{PARA CITAR ESTE ARTÍCULO / TO REFERENCE THIS ARTICLE / PARA CITAR ESTE ARTIGO /}

Vega, O.; De Marco, R.; Di Risio, C. (2015). Propiedades físicas y sensoriales de un pan fresco, con la adición de las enzimas lacasa, xilanasa y lipasa. Revista EIA, 12(24), julio-diciembre, pp. 87-100. [Online]. Disponible en: DOI: http:/dx.doi. org/10.14508/reia.2015.12.24.87-100 